\title{
Análisis histórico de la pérdida de humedales del Lago de Pátzcuaro, Michoacán, México
}

\author{
Historical analysis of loss from wetlands of Patzcuaro Lake, Michoacan, Mexico
}

\begin{abstract}
Lenin E. Medina-Orozco ${ }^{*}$, Norma E. García-Calderón², Felipe García Oliva ${ }^{3}$ y Elena Ikkonen ${ }^{4}$
Universidad Nacional Autónoma de México, Posgrado en Ciencias de la Tierra. Ciudad Universitaria. C.P 04510. Ciudad de México.

2 UMDI-FC-J. Universidad Nacional Autónoma de México. Boulevard Juriquilla 3001. C.P. 76230. Juriquilla, Querétaro, México.

3 IIES. Universidad Nacional Autónoma de México. Antigua Carretera a Pátzcuaro No.8701. Col. Ex Hacienda de San José de la Huerta. C.P. 58190, Morelia, Michoacán, México.

Instituto de Biología, Academia Rusa de las Ciencias de Karelia, Rusia.
\end{abstract}

\section{RESUMEN}

En el centro de México se presentan cuerpos de agua continentales como el lago de Pátzcuaro, con importantes ecosistemas de humedal con diversas funciones ecológicas. Actualmente, la presión sobre los recursos naturales en las cuencas del país, aceleran la pérdida de humedales. El objetivo del presente estudio fue evaluar la reducción que ha tenido el lago de Pátzcuaro en una serie de tiempo de 42 años, de 1972 a 2014 como un indicador de la pérdida de los humedales lacustres y su relación con dos componentes del balance hídrico; precipitación y evapotranspiración. El estudio se realizó utilizando técnicas de percepción remota (PR), basadas en detección de objetos sobre imágenes de satélite. Una interpretación manual de la pérdida y ganancia de los humedales fue realizada considerando el año de 1972 como la línea base del estudio. Los resultados indican una reducción sostenida de la superficie del lago, pasando de $111.7 \mathrm{~km}^{2}$ a $79.82 \mathrm{~km}^{2}$, a una tasa negativa de $0.72 \mathrm{~km}^{2}$ año-1. La longitud de la costa se modificó de $90.14 \mathrm{~km}$ en el año de 1972 a 71.84 km lineales en el año de 2014. Los cambios prominentes encontrados fueron: la pérdida del humedal de Tzurumútaro a partir de los años de 1980, la fusión de las islas Jarácuaro y La Pastora y su ulterior unificación con el área terrestre circundante del lago.

Palabras clave: escorrentía, hidrología, drenaje, erosión, humedad del suelo.

\section{ABSTRACT}

In central Mexico, continental water bodies such as the Patzcuaro Lake are present, with important wetland ecosystems and diverse ecological functions. Currently, pressure on natural resources in the country's watersheds accelerated the loss of wetlands. The aim of the present study was to evaluate the historical reduction of the Patzcuaro Lake in a time series of 42 years, from 1972 to 2014, as an indicator of the lacustrine wetlands loss and its relation with two components of the water balance: Precipitation and evapotranspiration. The study was carried out using remote sensing (PR) techniques, based on the detection of objects on satellite images. A manual interpretation of the loss and gain of wetlands was performed considering the year of 1972 as the study baseline. The results indicate a sustained reduction of the lake surface, from $111.7 \mathrm{~km}^{2}$ to $79.82 \mathrm{~km}^{2}$, at a negative rate of $0.72 \mathrm{~km}^{2} \mathrm{y}^{-1}$. The coast length was modified from $90.14 \mathrm{~km}$ in the year 1972 to 71.84 linear kilometers in the year 2014. The prominent changes found were: the loss of the Tzurumutaro wetland since the 1980s, the merger of the Jaracuaro and La Pastora islands and their subsequent unification with the surrounding land area of the lake.

Keywords: hydrology, drainage, erosion, soil moisture.

\section{INTRODUCCIÓN}

Los humedales son ecosistemas transicionales entre los terrestres y los acuáticos. El sistema más ampliamente utilizado para clasificar los humedales, fue propuesto por Cowardin et al. (1979), y considera tres atributos; (1) vegetación hidrófila predominante, (2) suelos hídricos, y (3) el sustrato puede no ser suelo (material orgánico), pero es saturado de agua o el nivel del agua es somero todo el año. De acuerdo con la Convención de Ramsar, a nivel global se calcula que la extensión mundial de los humedales disminuyó entre 64 y 71 \% en el siglo veinte (Gardner et al., 2015). En el caso de México, estimaciones recientes, sugieren que han desaparecido alrededor del $62 \%$ de sus humedales (Landgrave y Moreno-Casasola, 2012).

El monitoreo de humedales ribereños de las costas de los lagos, puede proveer de información valiosa para la investigación ecológica y el desarrollo de estrategias de conservación y mitigación ambiental de los cuerpos de agua (Frazier y Page, 2000; Rundquist et al., 2001; De Roeck, 2008); adicionalmente, los lagos pequeños, son considerados como centinelas de los cambios globales por su alta sensibilidad a los cambios ambientales (Adrian et al., 2009), y son sistemas integradores entre los cambios que ocurren en los sistemas terrestres circundantes (Williamson et al., 2008).

La Percepción Remota y los Sistemas de Información Geográfica (SIG) son herramientas poderosas para el monitoreo de los ecosistemas. Se ha documentado que el uso de imágenes Landsat, presentan resultados aceptables para delimitar humedales y cuerpos de agua continentales (De 
Roeck, 2008; Brown y Young, 2009). Sin embargo, uno de los problemas más frecuentes con los que se enfrenta la investigación en este ámbito, son los propios criterios de la percepción remota para su delimitación (Rundquist et al., 2001; Bagli y Soille, 2004; Brown y Young, 2009; Teferi et al., 2010). Actualmente existe una variedad de sistemas de clasificación de humedales y una variación de los criterios de delimitación de los cuerpos de agua (Cowardin, et al., 1979; Mitsch y Gosselink, 2000; Mitra et al., 2005; IPCC, 2006). A pesar de que actualmente existen herramientas poderosas para extraer la información satelital, se requieren de modelos detallados y dispendiosos en tiempo, principalmente para estudios de series de tiempo. Para solventar lo anterior se han propuesto técnicas de reconocimiento de patrones espaciales y concepción de imágenes correspondientes con la percepción humana (Ardila et al., 2005; Dahl y Bergeson, 2009; Blaschke, 2010; Estrada et al., 2013; Fickas et al., 2016). Cao et al. (2007), reportan que el mapeo de humedales con técnicas basadas en digitalización basadas en objetos, presentan valores razonables de precisión de $92.3 \%$ con respecto a las técnicas automatizadas, $73.6 \%$, basados en el valor de Kappa.

En el Centro de México existen lagos intermontanos de formación tectó-volcánica de gran importancia ecosistémica. Entre ellos, se encuentran la laguna de Chapala, la de Cuitzeo y el lago de Pátzcuaro, entre otros de menor magnitud. Pátzcuaro es el tercer lago más grande de México. Diversos estudios han manifestado cambios en el tamaño del espejo de agua y en su profundidad, tanto actuales como para periodos geológicos pasados relacionados con la propia evolución del Cinturón Volcánico Transmexicano (CVT) (Israde-Alcántara et al., 2005; Garduño-Monroy et al., 2009).

Estudios previos sugieren sensibilidad del lago de Pátzcuaro al cambio regional del clima (Gomez-Tagle et al., 2002) y se han documentado los efectos negativos que han tenido las actividades humanas sobre el lago, desde periodos precolombinos a la actualidad. Todo esto ha contribuido a la sedimentación del lago y a la disminución significativa del espejo de agua (Chacón-Torres et al., 1989; Chacón-Torres et al., 1992; Gómez Tagle, 1994; Gómez-Tagle et al., 2002; Fisher et al., 2003). Por lo anterior, resulta indispensable el seguimiento periódico de los cambios ocurridos en el lago de Pátzcuaro, que provean información y tendencias futuras.

El objetivo del presente estudio fue analizar una serie de tiempo de imágenes satelitales de 42 años (1972-2014), para evaluar si se presenta una pérdida sostenida de los humedales ribereños del lago de Pátzcuaro y su relación con el ambiente circundante en la cuenca de captación.

\section{MATERIALES Y MÉTODOS Área de estudio}

El Lago de Pátzcuaro se localiza en el centro de México en el Estado de Michoacán sobre el Cinturón Volcánico Transmexicano (CVT). El lago es de tipo endorréico de origen tecto-volcánico y litología dominada por derrames de lavas basálticas y andesíticas (Rosas et al., 1985; Garduño-Monroy et al., 2009), los suelos como consecuencia de la geología y variación altitudinal; son dominados por Andosoles en zonas altas y Luvisoles preferentemente en laderas de cerro (Gómez Tagle, 1994). El lago se encuentra entre los paralelos $19^{\circ} 32^{\prime}$ y $19^{\circ} 42^{\prime}$ latitud norte y los meridianos $101^{\circ} 45^{\prime}$ y $101^{\circ} 31^{\prime}$ longitud oeste, la altitud promedio del espejo de agua se ubica en los 2036 msnm. El vaso del Lago de Pátzcuaro presenta una orientación natural sur a norte de menor a mayor profundidad respectivamente (Figura 1). En el sur, la zona más somera del lago, se localizan los poblados ribereños de Erongarícuaro, Úrico, Arocutin, Jarácuaro, Tzurumútaro, Ihuatzio y las islas de Urandén, por el contrario; la zona más profunda corresponde al norte del lago, donde se encuentran los poblados de Tzintzunzan, Quiroga y Santa Fe de la Laguna (Chacón et al., 1989).
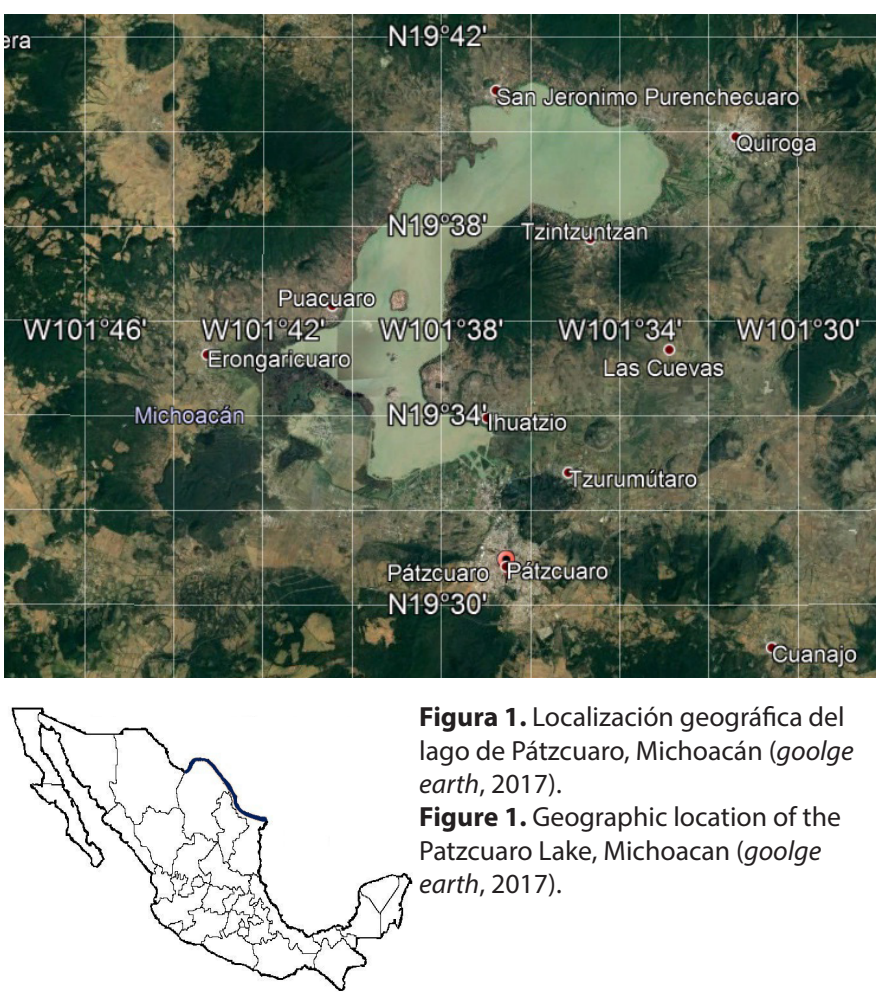

El clima zonal de acuerdo con las modificaciones de García (1987), es del tipo templado subhúmedo con lluvias en verano, se concentran de junio a septiembre con una precipitación total anual de $880 \mathrm{~mm}$ año ${ }^{-1}$, temperatura media anual de $16{ }^{\circ} \mathrm{C}$, el mes más cálido es junio [C (w2)(w)b(e)g] (Figura 2).

En el lago de Pátzcuaro la vegetación enraizada emergente, como la Typha y Schoenoplectus, es utilizada por artesanos de comunidades cercanas al lago para para la elaboración de artesanías (Hall, 2009), principalmente en la zona de Jarácuaro y Erongarícuaro (Fisher et al., 2003), así como la remoción de vegetación flotante y sumergida realizada frecuentemente, a cargo de una Comisión Gubernamental para mantener libres los accesos a las islas (Chacón et al., 1989). La población dentro de la cuenca es de 118,733 distribuida 


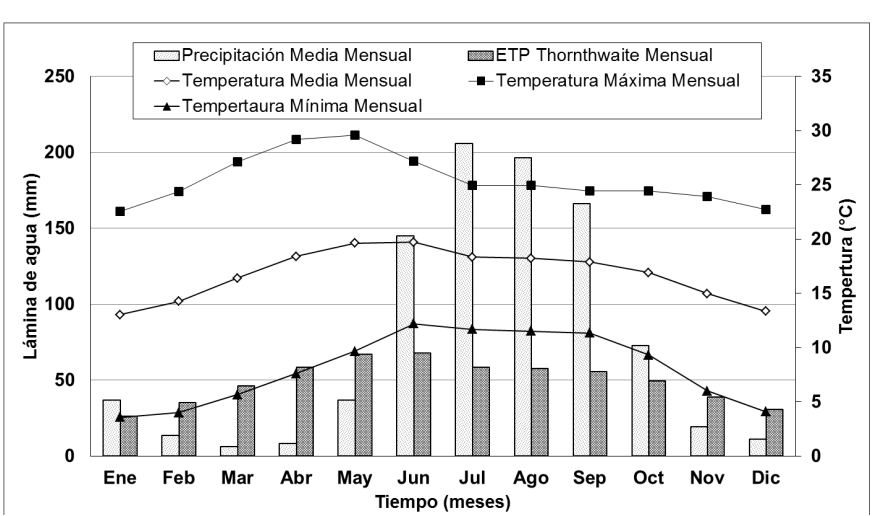

Figura 2. Climograma para la estación del lago de Pátzcuaro (1973-2010). Figure 2. Climatogram for the Patzcuaro Lake station (1973-2010).

en 24 comunidades, 17 ribereñas, cinco isleñas y dos de montaña, siendo las más pobladas Pátzcuaro, Tzintzuntzan y Erongarícuaro, respectivamente (SEPLADE, 2006).

\section{Colección de datos satelitales}

La delimitación del lago fue realizada para el presente estudio, sobre imágenes de satélite tipo landsat y Aster, con un total de 26 imágenes. Para las imágenes landsat, se colectaron un total de 23 imágenes. Las imágenes adquiridas corresponden a los tipos Multispectral Scanner (MSS) con resolución de 60 metros (1972 a 1981); Tematic Mapper (TM) con resolución de 30 metros (1982 a 1998) y Enhanced Tematic Mapper Plus (ETM+) con resolución de 30 metros de 1999 a 2009, así como tres imágenes ASTER para 2008, 2010 y 2014. Los años correspondientes a los años de 1974 a 1978, 1983 a 1985, 1987 y 1988, 2005 a 2007 y 2009 no tuvieron calidad suficiente para los propósitos de la investigación. La colección de imágenes fue obtenida del Centro de Observación y Ciencia de los Recursos de la Tierra (EROS) mediante la interfase: Glovis, por sus siglas en inglés (Global Visualization Viewer (http://glovis.usgs.gov/)). Las imágenes satelitales seleccionadas contemplaron los siguientes criterios; primero, que correspondieran a la época seca (enero-abril), y segundo que estuvieran libres de nubosidad sobre el área de estudio, ausencia de ruidos y fallas de los sensores.

Las imágenes cuentan con una ortocorrección de primer nivel L1T (Standar Terrain Correction, level 1T) que considera como base, un Modelo Digital del Terreno (DTM), generado a partir de bancos de nivel sobre la Tierra. Las imágenes contemplan el sistema WRS por sus siglas en inglés (World Reference System); en este sistema, el centro de la imagen se designa por una columna y una fila (columna 28 y fila 48), para este estudio. Mayores detalles pueden consultarse en (https://landsat.usgs.gov/landsat-processing-details) Las imágenes preferentemente corresponden a los periodos secos (febrero a abril), excepto en los casos donde no existió otra imagen disponible en la colección. El Sistema de Información Geográfica utilizado para el análisis de las imágenes satelitales fue ILWIS 3.2 Open.

\section{Ensayos preliminares para la delimitación del lago}

Para validar la corrección geométrica al nivel de $1 T$, se sobrepusieron las imágenes a elementos fijos de las cartas topográficas elaboradas por el Instituto Nacional de Geografía e Informática (INEGI); los elementos fueron carreteras y cruces de las mismas, vías de ferrocarril, centros poblacionales, entre otros. Se obtuvo una correspondencia razonablemente aceptable para la zona de estudio, por lo que no se consideró realizar una corrección geométrica adicional.

\section{Delimitación manual del lago}

En el presente trabajo se realizó una delimitación manual del cuerpo de agua, con base en el método sugerido por el Servicio de Pesca y Vida Silvestre de Estados Unidos, en sus procedimientos técnicos para el monitoreo de humedales (Dahl y Bergeson, 2009; Fickas et al., 2016). La digitalización visual se realizó sobre imágenes con compuestos de color verdadero (RGB: bandas 3, 2, 1) a una escala en pantalla de 1:50,000 teniendo en consideración los elementos de forma, color y textura de la costa del lago (Estrada et al., 2013; Fickas et al., 2016). En el presente estudio, la vegetación hidrófita no se consideró como base en la delimitación de los humedales, como ha sido sugerido por algunos autores (Rundquist et al., 2001; Teferi et al., 2010); lo anterior, con base en que la vegetación es sometida a constantes extracciones con fines artesanales y por otras actividades de interés económico (Fisher et al., 2003).

En este estudio se consideró cómo indicador de la pérdida o ganancia de humedales, al incremento o decremento del espejo de agua. En este estudio no se consideró el tipo de vegetación, debido a que es la principal fuente de variación en la delimitación de cuerpos de agua (Frazier y Page, 2000; De Roeck, 2008) y debido a que en esta región lacustre, la vegetación acuática emergente es extraída con fines artesanales (Fisher et al., 2003), adicionalmente durante los recorridos de campo se observaron quemas de vegetación acuática y pastoreo de ganado, por lo que se consideró que la vegetación hidrófita no sería un indicador razonable. Para caracterizar las tendencias de cambio en los humedales, se calcularon las pérdidas y ganancias, y el cambio neto en la serie de tiempo (1972 a 2014), de acuerdo con la propuesta metodológica de Fickas et al. (2016). El año de 1972, fue considerado como la línea base (superficie inicial) para evaluar las pérdidas o ganancias en el área de los humedales. La suma total de pérdidas-ganancias (considerando las islas), fue el cambio neto en los humedales para la serie de tiempo analizada, en función de la metodología de Fickas et al. (2016) y con base en la clasificación de los humedales propuesta por Cowardin et al. (1979), siendo del tipo de humedal lacustre emergente, donde es temporalmente inundado y regulado por la estacionalidad de las lluvias.

\section{Base de datos climatológicos}

Los datos climatológicos de temperatura y precipitación diarias, fueron obtenidos de la base de información de 
la Red de Datos Climatológicos de México (CLICOM) compilados por la Comisión Nacional del Agua (CONAGUA, 2003). Los datos utilizados para el balance de agua fueron: precipitación y temperaturas promedio, temperaturas máximas promedios y evaporación de tanque $A$, promedio diarios.

Para estimar el balance hidrológico de la cuenca, se utilizó una familia de nueve estaciones: Pátzcuaro (16087), Los Chorros del Varal (16072), Taretan (16127), Ingenio San Sebastián (16053), Los Limones, Los Reyes (16073), Jicalan, Uruapan (16056), Uruapan (16138), Peribán (16088) y Barranca de Cupatitzio (16212). Se obtuvo la ecuación de regresión para la precipitación en función de la altitud (Ecuación 1).

Precipitación $(\mathrm{mm})=0.8994 x+42.85 ; R^{2}=0.9264$

Ecuación (1)

\section{Precipitación en la cuenca}

La cuenca de Pátzcuaro tiene una superficie total de $918 \mathrm{~km}^{2}$. Los rangos altitudinales y su superficie se describen a continuación: de 2037 a 2038 msnm el área es de 114.7 km² (área promedio del espejo de agua y zonas de inundación), de 2039-2440 msnm (583.9 km²), de 2441-2640 msnm (115.3 $\left.\mathrm{km}^{2}\right)$, de 2641-3240 msnm (102 km²), y de 3241-3420 msnm $\left(2.1 \mathrm{~km}^{2}\right)\left(803.3 \mathrm{~km}^{2}\right.$ área total terrestre). Con los datos anteriores y la ecuación 1 , se obtuvo la precipitación ponderada total de la cuenca.

\section{Evapotranspiración de la cuenca}

Esta variable se estimó por dos modelos; el primero se aplicó a la superficie terrestre de la cuenca, para el periodo de 1972 a 2010. El modelo de evapotranspiración fue el de Thornthwaite que presenta resultados razonables en cuencas cerradas. El modelo permite estimar la evapotranspiración potencial de un lugar para cada mes del año a partir de los parámetros básicos ETp (evaporación potencial media del mes) e I (índice de calor mensual):

$E T p=1.6(10 t / I)^{a}$

Ecuación (2)

Donde la ETp = evapotranspiración potencial media diaria del mes $\left(\mathrm{mm} \mathrm{d}^{-1}\right)$, para meses de 30 días y 12 horas de luz diaria; $\mathrm{t}=$ temperatura media diaria del mes en materia $(\circ \mathrm{C})$ en la zona de estudio. Calculada a partir de las medias diarias; I = índice de calor anual. Calculado como suma de los índices de calor mensual (I) de los 12 meses del año y $a=$ coeficiente experimental de ajuste (Kairu, 1991; Rosenberry et al., 2007):

$a=\left.\left(675 \cdot 10^{-9}\right)\right|^{3}-\left.\left(771 \cdot 10^{-7}\right)\right|^{2}-\left(1972 \cdot 10^{-5}\right) \mid+0.4924$

Ecuación (3)

En el segundo modelo, se realizó un ajuste de la ecuación 3 para incluir el efecto de la vegetación acuática en la evapotranspiración del lago (Yu y Fassman, 1998; Xu y Singh, 2001; Martínez et al., 2005).

$E T=0.7 \times$ Evaporación del tanque tipo A

Ecuación (4)

\section{RESULTADOS Y DISCUSIÓN}

Los resultados sugieren que el lago de Pátzcuaro ha perdido humedales lacustres de manera sostenida para los 42 años analizados (Figura 3). Se han presentado cambios en la morfología y en la longitud de la costa del lago. En igual forma, se presentaron nuevos elementos terrestres a manera de islotes, así como la fusión de las islas de Jarácuaro y La Pastora, primero entre sí y ulteriormente una fusión con la zona terrestre para dejar de existir como islas.

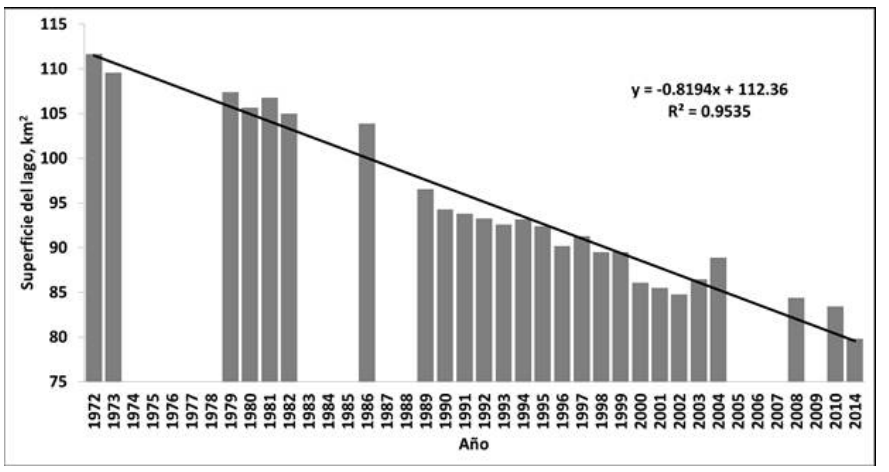

Figura 3. Pérdida en superficie $\left(\mathrm{km}^{2}\right)$ de los humedales en el lago de Pátzcuaro para una serie de tiempo de 42 años (de 1972 a 2014).

Figure 3. Loss of wetlands $\left(\mathrm{km}^{2}\right)$ from the Patzcuaro Lake in a 42-year period (1972-2014).

\section{Pérdida de humedales}

Para la serie de tiempo estudiada el lago presentó una reducción significativa en el tamaño de su superficie, interpretada como pérdida de humedales, cambiando de $111.7 \mathrm{~km}^{2}$ en 1972 a $72.2 \mathrm{~km}^{2}$ en el año de 2014 . La reducción neta para un lapso de 42 años fue de $39.5 \mathrm{~km}^{2}$ (35.3\%). La tasa promedio de pérdida de humedales fue de $0.94 \mathrm{~km}^{2}$ año-1 (Figuras 3 y 4). Tres periodos acentúan la pérdida de los humedales: el primero se presentó entre 1972 y 1980 con una pérdida de superficie de $6 \mathrm{~km}^{2}$ con respecto a la línea base $\left(111.7 \mathrm{~km}^{2}\right)$; otro periodo importante ocurrió entre 1986 a 1989, con una reducción de 9.6 km² un tercero entre 2005 y 2009 que causó una reducción del área superficial de 4.5 $\mathrm{km}^{2}$. Estos tres periodos, por si solos, sumaron el $71.5 \%$ de la pérdida de superficie de humedales (Figura 3 ).

La longitud de la costa sufrió un cambio al pasar de 90.144 km en el año de 1972, a 71.84 km en 2014; es decir, se redujo $18.304 \mathrm{~km}$. La reducción en la longitud de la costa se relaciona directamente con dos cambios significativos en la morfología del lago: la primera es producto de la desecación de una zona somera localizada al pie del cerro el Vado ubicado entre la ciudad de Pátzcuaro y Tzurumútaro (Figura 4, letra a), donde se perdieron $7.6 \mathrm{~km}^{2}$, que representan el $8.5 \%$ con respecto a la superficie inicial que se tenía en 1972. La segunda fue causada por la fusión entre las islas de Jarácuaro-La Pastora y su posterior unión con la zona continental.

En 1972 Jarácuaro y La Pastora se presentaban como dos islas, y ulteriormente fueron reduciendo su distancia hasta encontrarse separadas únicamente por un dren artificial en dirección norte-sur en el año de 1989. La fusión de estos dos elementos se presenta a partir de 1991. Actualmente 


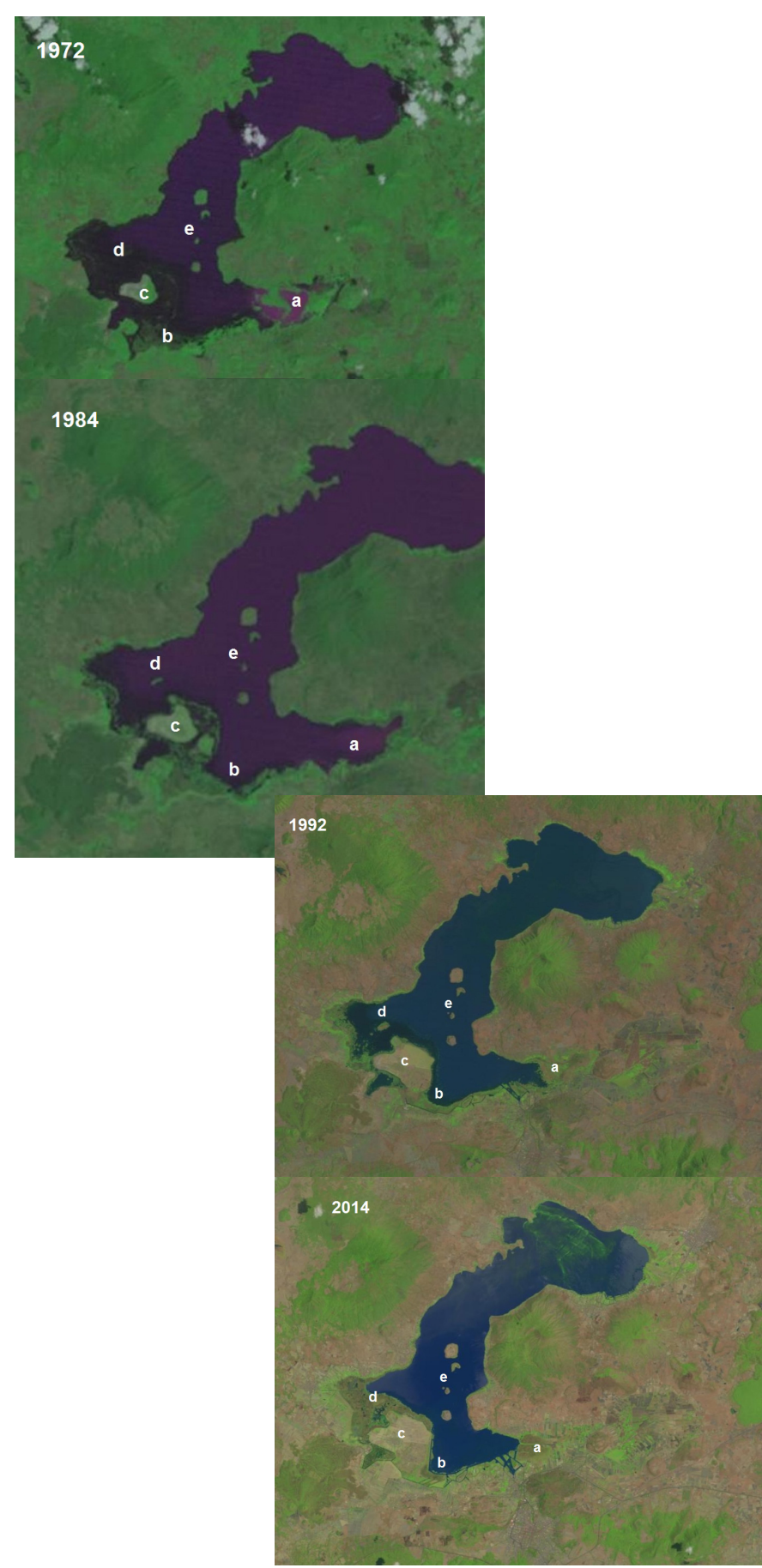

Figura 4. Morfología y elementos terrestres en el lago de Pátzcuaro. Letras $a, b, c$, d y e representan las zonas donde ocurrieron los principales cambios, primordialmente entre 1972 y 2014 (USGS Landlook).

Figure 4. Morphology and terrestrial elements in Patzcuaro Lake. letters a, $b, c, d$ and e, represent the areas where the main changes occurred, primarily between 1972 and 2014 (USGS Landlook).

esta zona se conoce como la isla de Jarácuaro y se omitió el nombre de La Pastora. La isla fue incrementando su superficie entre 1972 y 2014 en 3.4 veces su tamaño para la línea base.

\section{Cambios morfológicos al interior del lago}

Por otro lado, se presentaron evidentes cambios al interior del lago a partir del año de 1979, principalmente en la porción sur. Estos cambios se mostraron como nuevos elementos emergidos a manera de islotes frente al poblado de Uricho (Figura 4, letra d). Para el año 2014 este islote presenta dimensiones de $1035 \mathrm{~m}$ en longitud, ancho máximo de 311 $\mathrm{m}$, y superficie de $0.3 \mathrm{~km}^{2}$. La zona media del lago, aparece a partir de 1989 un pequeño islote con área de $0.02 \mathrm{~km}^{2}$, próximo a la isla de La Tecuena (Figura 4, letra e). Estos cuerpos terrestres de reciente exposición, no tienen nombre oficial en la cartografía topográfica reciente del INEGI.

\section{Balance hidrológico}

La precipitación promedio anual para el periodo de estudio fue de $904.8 \pm 110.5 \mathrm{~mm}$, con precipitaciones máximas históricas de $1231.7 \mathrm{~mm}$ y mínima de $755 \mathrm{~mm}$, correspondientes a los años de 2004 y 2005 respectivamente. En términos de volumen de agua precipitada, el promedio anual para la serie de tiempo fue de $1217.8 \mathrm{Mm}^{3}$, distribuido de la siguiente manera: $103.8 \mathrm{Mm}^{3}$ (8.4\%) sobre el lago, $1114 \mathrm{Mm}^{3}$ (91.6\%) en la cuenca de captación. Por el contrario, la evapotranspiración (Etp) promedio anual para la serie de tiempo fue de $1583 \mathrm{~mm}\left(581.7 \mathrm{Mm}^{3}\right)$, distribuido de la siguiente manera: sobre el lago, $990 \mathrm{~mm}\left(113.6 \mathrm{Mm}^{3}\right)$ y en la cuenca de captación, $593 \mathrm{~mm}\left(468.1 \mathrm{Mm}^{3}\right)$. El balance entre los dos componentes del ciclo hidrológico, sugieren que la evapotranspiración en la cuenca fue inferior a la precipitación para la serie de tiempo. Lo anterior significa que existe en términos globales, un excedente de agua en la cuenca de $645.9 \mathrm{Mm}^{3}$ para abastecer el cuerpo de agua. De lo anterior, podemos deducir que el balance de agua en la cuenca es positivo y no es suficiente para explicar la reducción actual de la superficie del lago en función de estos componentes del balance hídrico. Adicionalmente, los resultados indicaron que las temperaturas en la cuenca de Pátzcuaro han presentado un incremento (Figura 5), siendo las temperaturas máximas las que presentan aumentos significativos $\left(R^{2}=0.7517\right)$, por ejemplo, para la década de los $70^{\prime} \mathrm{s}$ las temperaturas máximas eran de $23.5^{\circ} \mathrm{C}$ en promedio, de $25.1,26.7$ y $27^{\circ} \mathrm{C}$ para los años de 1980, 1990 y 2000, respectivamente. Los resultados pueden

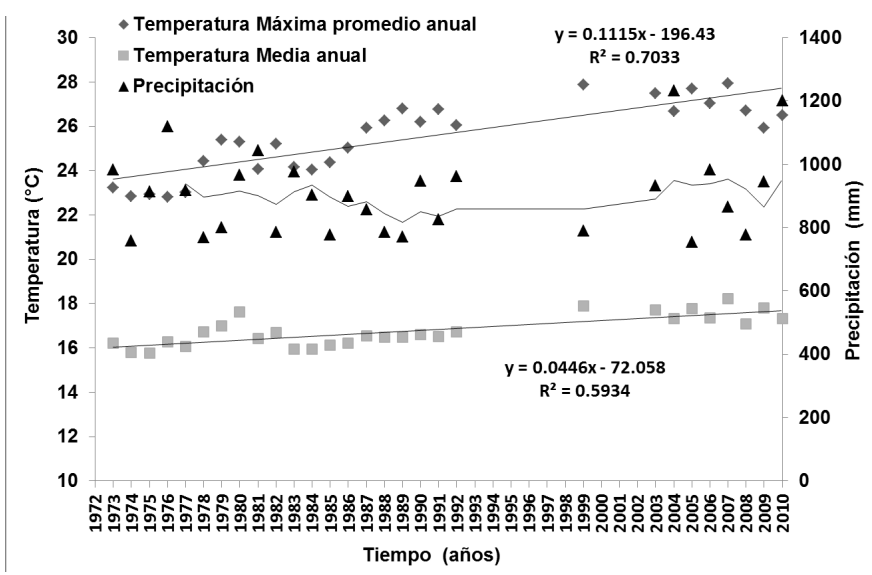

Figura 5. Tendencia de la temperatura atmosférica y la precipitación en la cuenca del lago de Pátzcuaro (media móvil de cinco años).

Figure 5. Trend of atmospheric temperature and precipitation in the Patzcuaro lake basin (five-year mobile average). 
interpretarse como una respuesta al ambiente circundante de la cuenca. El incremento de la temperatura modifica las tasas de evapotranspiración (Lofgren et al., 2011).

\section{Cobertura vegetal y uso del suelo}

Las coberturas vegetales y usos del suelo (CVUS) son dominadas por bosque de pino, bosque de encino o mixto de encino pino, y agricultura de temporal principalmente (Tabla 1). Las CVUS no presentaron cambios significativos en las coberturas de los bosques solo incrementaron en un 2.5 $\%(6,321 \mathrm{ha})$, pero se observa una tendencia en la reducción en las áreas dedicadas a la agricultura, pasando a ser coberturas de matorral y pastizales.

Tabla 1. Cobertura vegetal y usos del suelo (CVUS) para un periodo de 10 años en la cuenca de Pátzcuaro, Michoacán, México.

Table 1. Vegetation cover and land uses (VCLU) for a 10 year-period in the Patzcuaro basin, Michoacan, Mexico.

\begin{tabular}{lcccccc}
\hline \multirow{2}{*}{ Año } & \multicolumn{2}{c}{1986} & \multicolumn{2}{c}{1996} & \multicolumn{2}{c}{2005} \\
\cline { 2 - 7 } CVus & ha & $\%$ & ha & $\%$ & ha & $\%$ \\
\hline Bosques $^{\text {a }}$ & 28,019 & 30.0 & 34,340 & 32.5 & 33,670 & 22.3 \\
Uso agrícola $_{\text {Matorral / Pastizal }}$ & 38,141 & 40.8 & 23,714 & 22.4 & 20,530 & 36.6 \\
Otros usos & 13,745 & 14.7 & 33,240 & 31.4 & 19,590 & 21.3 \\
Total & 13,555 & 14.5 & 14,503 & 13.7 & 18,140 & 19.7 \\
\hline
\end{tabular}

a incluye bosque de pino, pino encino mixto y encino pino mixto. * No incluye el cuerpo de agua, ${ }^{* *}$ incluye el cuerpo de agua. Gómez-Tagle, 1994; Álvarez Icaza et al., 1996; Tapia et al., 2005. Estadísticamente no se presentaron diferencias en los CVUS (Tukey, $a=0.05, p=0.859$ ).

A nivel global se tienen reportes de que los humedales han disminuido en superficie entre un $64 \%$ y $71 \%$ en el siglo veinte (Gardner et al., 2015). En referencia a los humedales lacustres, diversos autores sugieren que han sido destruidos o transformados a tasas aceleradas por cambios de uso de suelo. Para ilustrar lo anterior, en China se estima que se han perdido en un lapso de 50 años el $21.6 \%$ los humedales (An et al., 2007). Situaciones similares se presentan en Australia y África (Kingsford y Thomas, 2004; Balirwa, 1995). En el caso de México estimaciones recientes sugieren que han desaparecido alrededor del $62 \%$ de sus humedales (Landgrave y Moreno-Casasola, 2012). En este mismo sentido, el lago de Pátzcuaro no sería la excepción, sin embargo, las posibles causales de la reducción de los humedales lacustres pueden deberse a causas diversas.

Los resultados sugieren que las entradas de agua vía precipitación son mayores que las pérdidas por evapotranspiración y coincide con lo reportado por otros autores (Chacón-Torres et al., 1989; Gómez-Tagle et al., 2002; BernalBrooks et al., 2002). La precipitación se ha mantenido relativamente constante y se observó que la temperatura media no presentó incrementos significativos. A nivel global se ha encontrado que la precipitación y la evapotranspiración son suficientes para explicar los cambios de pequeños lagos en cuencas cerradas (Jones et al., 2001). Sin embargo, las temperaturas promedio máximas presentaron incrementos significativos en la serie de tiempo y en este sentido, Williamson et al. (2008) consideran a la temperatura como una variable de respuesta a los cambios ambientales circundantes de los lagos, que pueden incrementar las tasas de evapotranspiración, como ha sido estudiado por Blanken et al. (2011) en el lago Superior de Norteamérica. Desafortunadamente, los modelos disponibles para calcular la evapotranspiración a nivel de cuenca y la poca instrumentación de la cuenca de Pátzcuaro, solo permiten estimar este parámetro a partir de modelos teóricos, como en el presente estudio, basados en las temperaturas promedio, sin que sea posible, integrarlos con las temperaturas máximas (Lofgren et al., 2011). Otros autores han sugerido una sensibilidad en el nivel y volumen del agua del lago de Pátzcuaro, al cambio regional del clima (Gomez-Tagle et al., 2002), por lo anterior es necesario, profundizar en estudios de balance hidrológicos sensibles a cambios en la amplitud de las temperaturas y de cambio climático.

El cambio de uso del suelo, es un elemento modificador de las condiciones ambientales de los lagos y del almacén de agua de los mismos (Williamson et al., 2008; Blanken et al., 2011; Lofgren et al., 2011). De acuerdo con la literatura, los CVUS son estables entre 1986 y 2005 (Tabla 1), sin embargo, es necesario actualizar los CVUS de la cuenca y separar las áreas de humedales, por ser un tipo de vegetación con alta evapotranspiración (Sánchez-Carrillo et al., 2004). Jones et al. (2001) encontraron evidencia del efecto de los cambios de la vegetación y usos de suelo regionales, sobre tres pequeños lagos, más allá de sus cuencas circundantes. Se han documentado efectos negativos que han tenido sobre el lago las actividades humanas, desde periodos precolombinos a la actualidad, que han contribuido a la sedimentación del lago y la disminución significativa del espejo de agua (Chacón-Torres et al., 1989; Gómez Tagle, 1994; Gómez-Tagle et al., 2002; Fisher et al., 2003). Por lo anterior, es importante explorar y ampliar los estudios de la erosión como lo sugieren Tapia et al. (2005), y poder explicar la pérdida de humedales. Asimismo, se deben incrementar los estudios sobre el uso consuntivo de agua de los manantiales de la cuenca de Pátzcuaro que podrían estar siendo sobreutilizados (Bischoff et al., 2004). Es importante incluir estudios sobre la dinámica poblacional de la cuenca, si bien, algunos estudios sugieren que la población en las últimas décadas se ha mantenido estable, explicado principalmente por los procesos migratorios de la comunidad P'urepecha hacia los Estados Unidos de Norteamérica, principalmente de los jóvenes quienes a diferencia de los adultos emigraban de forma definitiva (Enrique, 2017). Aspectos culturales de manejo del agua como son la pesca, el uso de la vegetación emergente (chuspata) para elaboración de artesanías (Fisher et al., 2003), la ganadería ribereña de ganado bovino, recientemente el cultivo de aguacate, y la erosión del suelo, deben de ser componentes para estudios posteriores que permitan comprender los procesos de la pérdida en el tamaño del espejo de agua de este importante lago de México. 


\section{CONCLUSIONES}

La pérdida de superficie del lago y de sus humedales es evidente para la serie de tiempo estudiada, perdiendo $31.88 \mathrm{~km}^{2}$ entre 1972 y 2014. Los cambios más prominentes identificados consistieron en la desecación del humedal de Tzurumútaro, la fusión de las Islas Jarácuaro y la Pastora, y la aparición de nuevos elementos terrestres al interior del lago como islotes. El balance de agua para los componentes precipitación y evapotranspiración, no fueron suficientes para explicar la pérdida de los humedales lacustres ni la cobertura vegetal. Por lo anterior, es necesario realizar estudios y explorar modelos que permitan medir la evapotranspiración con base en las temperaturas máximas y procesos de sedimentación, uso consuntivo del agua y de cambios de cobertura vegetal a mayor escala.

\section{AGRADECIMIENTOS}

El primer autor agradece el apoyo al Consejo Nacional de Ciencia y Tecnología (CONACYT) por la beca otorgada número 17264 para cursar el doctorado en Ciencias de la Tierra dentro del programa de la Universidad Nacional Autónoma de México. Así como al proyecto: Focos rojos de gases con efecto de invernadero en México: Estructura y funcionamiento de los suelos saturados de humedad. Semarnat-CONACyT 23489 y al proyecto PAPIIT-IN224410. Se agradecen las observaciones de parte de los revisores para mejorar sustancialmente el presente documento.

\section{REFERENCIAS}

Adrian, R., O'Reilly, C. M., Zagarese, H., SBaines, B., Hessen, D. O., Kellerf, W., Livingstone, D. M., Sommaruga, R., Straile, D., Van Donk, E., Weyhenmeyer, G. A. y Winder, M. 2009. Lakes as sentinels of climate change. Limnology and Oceanography. 54(6): 2283-2297.

An, S., Li, H., Guan, B., Zhou, C., Wang, Z., Deng, Z., Zhi, Y., Liu, Y., Xu, C., Fang, S., Jiang, J. y Li, H. 2007. China's natural wetlands: past problems, current status, and future challenges. Ambio. $36(4): 335-342$.

Ardila, L. J. P., Espejo, O. J., V. y J. L. Herrera, E. 2005. Validación de una metodología de clasificación de imágenes satelitales en un entorno orientado a objetos. Ingeniería. 10(1): 61-69.

Bagli, S. y Pierre, S. 2004. Automatic delineation of shoreline and lake boundaries from Landsat satellite images, Proceedings of initial ECO-IMAGINE GI and GIS for Integrated Coastal Management, Seville 13th-15th May 2004.

Balirwa, J. S. 1995. The Lake Victoria environment: Its fisheries and wetlands - a review. Wetlands Ecology and Management. 3: 209-224.

Bernal-Brooks, F. W., Gómez-Tagle, R. A. y Alcocer, J. 2002. Lake Patzcuaro (Mexico): a controversy about the ecosystem water regime approached by field references, climatic variables, and GIS, Hydrobiologia. 467: 187-197.

Bischoff, J. L., Israde-Alcantara, I., Garduño-Monroy, V.H. y Wayne, C. 2004. The springs of Lake Pátzcuaro: chemistry, salt-balance, and implications for the water balance of the lake. Applied Geochemistry. 19(11): 1827-1835.

Blanken. P. D., Spence, C., Hedstrom, N. y Lenters, J.D. 2011. Evaporation from Lake Superior: 1. Physical controls and processes. Journal of Great Lakes Research. 37(4): 707-716.

Blaschke, T. 2010. Object based image analysis for remote sensing. ISPRS Journal of Photogrammetry and Remote Sensing. 65(1): 2-16.

Brown, L. y Young, K. L. 2009. Assessment of the three mapping techniques of delineate lakes and ponds in a canadian high artic wetland complex. Artic. 59(3): 283-293.

Cao, M., Liu, G. y Zhang, X. 2007. An object-oriented approach to map wetland vegetation: a case study of Yellow river delta. Geoscience and Remote Sensing Symposium, 2007. IGARSS 2007. IEEE International.

Cowardin, L. M., Carter, V., Golet, F. C. y LaRoe, E. T. 1979. Classification of Wetlands and Deepwater Habitats of the United States. U.S. Department of the Interior. Fish and Wildlife Service. Office of Biological Services. USGS. 79 p. Washington, D.C.

Chacón, T. A., Ross, L. G. y Beveridge, M. C. M. 1989. Lake Patzcuaro, Mexico: results of the new morphometric study and its implications for productivity assessments. Hydrobiologia. 184: 125-132.

Chacón, T. A., Ross, G. L., Beveridge, M. C. M. y Watson, A. 1992. The application of SPOT multiespectral imagery for the assessment of water quality in lake Patzcuaro, Mexico. International Journal of Remote Sensing. 13(4): 587-603.

Comisión Nacional del Agua. 2003. CLICOM Red de datos de estaciones Climatológicas de México, México [cd-rom].

Dahl, T.E. y Bergeson, M. T. 2009. Technical procedures for conducting status and trends of the Nation's wetlands. U. S. Fish and Wildlife Service, Division of Habitat and Resource Conservation, 74 p. Washington, D.C.

Estrada, L. F., Barba, M. E., y Ramos, R. R. 2013. Cobertura temporal de los humedales en la cuenca del Usumacinta, Balancán, Tabasco, México. Universidad y Ciencia. 29(2): 141-151.

Fickas, K. C., Cohen, W. B. y Yang, Z. 2016. Landsat-based monitoring of annual wetland change in the Willamette Valley of Oregon, USA from 1972 to 2012. Wetlands Ecology and Management. 24:73-92.

Fisher, C. T., Pollar. H. P., Israde-Alcántara, I., Garduño-Monroy, V. H. y Banerjee, S. K. 2003. A reexamination of human induced environmental change in the lake pátzcuaro basin, Michoacán, Mexico. Anthropology. 100(8): 4957-4962.

Frazier, P. S. y Page, K. J. 2000. Water body detection and delineation with Landsat TM data. Photogrammetric Engineering \& Remote Sensing. 66 (12): 1461-1467.

García, De M. E. 1987. Modificaciones al sistema de clasificación climática de Köppen (para adaptarlo a las condiciones de la República Mexicana). Cuarta Edición. México.

Gardner, R.C., Barchiesi, S., Beltrame, C., Finlayson, C.M., Galewski, T., Harrison, I., Paganini, M., Perennou, C., Pritchard, D.E., Rosenqvist, A. y Walpole, M. 2015. State of the world's wetlands and their services to people: a compilation of recent analyses. Ramsar Briefing Note no. 7. Gland, Switzerland: Ramsar Convention Secretariat.

Garduño-Monroy, V. H., Chávez-Hernández, J., AguirreGonzález, J., Vázquez-Rosas, R., Mijares-Arellano, H., IsradeAlcántara, I., Hernández-Madrigal, V.M., Rodríguez-Pascua, M. A. y Pérez-López, Y R. 2009. Zonificación de periodos naturales de oscilación superficial en la ciudad de Pátzcuaro, Mich., México, con base en microtremores y estudios de paleosismología. Revista Mexicana de Ciencias Geológicas. 6 (3): 623-637. 
Google earth. 2017. Google inc. https://www.google.com.co/ maps. Recuperado 02 de febrero de 2017.

Gomez-Tagle, CH. A., Bernal-Brooks, F.W. y Alcocer, J. 2002. Sensivity of Mexican water bodies to regional climatic change: three study alternatives applied to remote sensed data of lake Patzcuaro, Hydrobiologia. 467: 169-176.

Gómez-Tagle, R. 1994. Tres niveles de erosión en la cuenca de Pátzcuaro, Michoacán: como base para acciones y obras de conservación. Folleto técnico $\mathrm{N}^{\circ} 26$. Instituto Nacional de Investigaciones Forestales y Agropecuarias. Secretaria de Agricultura y Recursos Hidráulicos.

Hall, S. J. 2009. Cultural disturbances and local ecological knowledge mediate cattail (Typha domingensis) invasion in lake Pátzcuaro, México. Human Ecology. 37:241-249. doi: 10.1007/s10745-009-9228-3.

IPCC. 2006. Guidelines for national greenhouse inventories, vol. 4. Agriculture, forestry and other uses (AFLOLU). Institute for Global Environmental Strategies, Hayama, Japan.

Israde-Alcántara, I., Garduño-Monroy, V. H., Fisher, C. T., Pollar, H. P. y Rodríguez-Pascua, M. A. 2005. Lake level change, climate, and the impact of natural events: the role of seismic and volcanic events in the formation of the Lake Patzcuaro Basin, Michoacan, Mexico. Quaternary International. 135: 35-46.

Kairu, E. N. 1991. A review of methods for estimating evapotranspiration: particularity those that utilize Remote Sensing. GeoJournal. 25(4): 371-376.

Kingsford, R. T. y Thomas, R. F. 2004. Destruction of wetlands and waterbird populations by dams and irrigation on the Murrumbidgee River in arid Australia. Environmental Management. 34(3). 383-396.

Lofgren, B. M., Hunter, T. S. y Wilbarger, J. 2011. Effects of using air temperature as a proxy for potential evapotranspiration in climate change scenarios of Great Lakes basin hydrology. Journal of Great Lakes Research. 37(4): 744-75.

Jones, R. N., McMahon., T. A. y Bowler, J. M. 2001. Modelling historical lake levels and recent climate change at three closed lakes, Western Victoria, Australia (c.1840-1990). Journal of Hydrology. 246(1-4):159-180. doi: 10.1016/S00221694(01)00369-9.

Landgrave, R.y Moreno-Casasola, P. 2012. Evaluación cuantitativa de la pérdida de humedales en México. Investigación ambiental. 4 (1): 19-35.

Martínez, R., Boueri, M. A. y Escalona, P. 2005. Correlación entre la evaporación en tina y la evapotranspiración de referencia en cinco estaciones climatológicas de Venezuela. Bioagro. 17 (2): 79-83.

Mitra, S., Wassmann., R. y Vlek, P. L. G. 2005. An appraisal of global wetland area and its organic carbon stock. Current Science. 88 (1): 25-35.
Mitsch, W. y Gooselink, J G. 2000. Wetland Biogeochemistry. En: Wetlands. Mitsch, W. J and G. Gooselink (eds). Chapter 6. $3^{\text {th }}$ edition. John Wiley and Sons. USA.

Rosas, I., Mazari, M., Saavedra, J. y Baez, A. P. 1985. Benthic organisms as indicators of water quality in lake Patzcuaro, Mexico. Water, Air, and Soil Pollution. 25: 401-414.

Rosenberry, D. O., Winter, T. C., Buso, D. C. y Likens, G. E. 2007. Comparison of 15 evaporation methods applied to the small mountain lake in the northeastern USA. Journal of Hydrology. 340: 149-166.

Rundquist, D. C., Narulamani, S. y Narayanan, R. M. 2001. A review wetlands remote sensings and defining new considerations. Remote sensings reviews. 20: 207-226.

Sánchez-Carrillo, S., Angeler, D. G., Sánchez-Andrés, R., AlvarezCobelas, M., y Garatuza-Payán, J. 2004. Evapotranspiration in semi-arid wetlands: relationships between inundation and the macrophyte - cover: open-water ratio. Advances in Water Resources. 27 (6): 643-655.

SEPLADE. 2006. Carpeta Municipal de Pátzcuaro, México, Secretaria de Planeación y Desarrollo Estatal, Gobierno del Estado de Michoacán.

Tapia, C. E. 2017. Las migraciones michoacanas: tradiciones y prácticas migratorias. 91-119 pp. En: (C. Heller R. y E. Coral G. Coords.). El Impacto Sociocultural del Fenómeno Migratorio en Michoacán. Pátzcuaro, Michoacán. México.

Tapia, V. L. M., Tiscareño, L. M., Chavez-Leon, I. G., Fernandez, V. y Reyes, M. L. 2005. Modeling runoff and erosion in the lake of Patzcuaro watershed, Michoacan, Mexico. 160-188 pp. En: Modeling and Remote Sensing Applied to Agriculture (U.S. and Mexico). Richardson C. W (ed). USDA Agriculture Research Service. USA.

Teferi, E., Uhlenbrook, S., Bewket, W., Wenninger, W. y Simane, B. 2010. The use of the remote sensing to quantify wetland loss in the Choke Mountain range, Upper blue Nile basin, Ethiopia. Hydrology and Earth System Sciences Discussions. 7: 6243-6284.

Tiscareño, L. M., Baez, G. A. D., Velazquez, V. M., Potter, K. N., Stone, J. J., Tapia, V M. y Claveran, R. A. 1999. Agricultural research for watershed restoration in central Mexico. Journal of Soil and Water Conservation. 54: 686-692.

Williamson, C. E., Dodds, W., Kratz, T. K., y Palmer, M. A. 2008. Lakes and streams as sentinels of environmental change in terrestrial and atmospheric processes. Frontiers in Ecology and Environment. 6(5):247-254.

Yu, S. L. y Fassman, E. A. 1998. Hydrologic budget for a wetland system. Virginia Transportation Research Council. Charlottesville, Virginia. USA.

Xu, C. Y. y Singh, V.P. 2001. Evaluation and generalization of temperature-based methods for calculating evaporation. Hydrological Processes. 15: 305-319. 